\title{
Glycosylated Siglec-6 Expression in Syncytiotrophoblast-derived Extracellular Vesicles from Preeclampsia Placentas
}

Toluwalase Awoyemi ${ }^{1 *}$, Dionne Tannetta ${ }^{2 *}$, Wei Zhang ${ }^{1}$, Neva Kandzija ${ }^{1}$, Carolina MottaMejia $^{1}$, Roman Fischer ${ }^{3}$, Raphael Heilig ${ }^{3}$, Sina Raiss ${ }^{1}$, Christopher Redman ${ }^{1} \&$ Manu Vatish ${ }^{1}$

* Equal Contribution

${ }^{1}$ Nuffield Department of Women's \& Reproductive Health, University of Oxford, Oxford, United Kingdom

${ }^{2}$ School of Pharmacy, University of Reading, Reading, United Kingdom

${ }^{3}$ Discovery Proteomics Facility, Target Discovery Institute, Nuffield Department of Medicine, University of Oxford, Oxford, OX3 7FZ, United Kingdom

*Corresponding author: Dr Manu Vatish, MBBCh, BA (Hons), DPhil, MA, FMRCOG

Address: Nuffield Department of Women's and Reproductive Health, University of Oxford, Women’s Centre, John Radcliffe Hospital, Oxford OX3 9DU, United Kingdom 
Telephone: $+44(0) 1865221009$

Fax: $+44(0) 1865769141$

Email: manu.vatish@wrh.ox.ac.uk

\section{ABSTRACT}

\section{INTRODUCTION}

Preeclampsia (PE) is associated with an exaggerated maternal systemic inflammatory response. Throughout gestation, the placenta releases extracellular vesicles through the syncytiotrophoblast layer (STB) into the maternal circulation and this is increased in PE. Expression of Siglec-6, a transmembrane receptor of molecular weight 50KDa, is upregulated in PE placental tissue.

\section{METHODS}

Here we investigated respective abundance of Siglec-6 in PE -and normal pregnancy- (NP) derived placental lysates (PL) and syncytiotrophoblast-derived extracellular vesicles (STBEV). STBEV from PE and NP placentas were isolated through dual-lobe placental perfusion and serial ultracentrifugation. Siglec-6 was characterized by immunohistochemistry, immunoblotting, mass spectrometry (MS), and deglycosylation.

\section{RESULTS}

Immunoblotting revealed the expected Siglec-6 (50KDa) band present in both PE and NP PL, however an additional heavier band was observed at 70KDa only in PE PL, but not in NP. When interrogating STBEV we saw an absence of the expected 50KDa band but the 70KDa was present predominantly only in the PE STBEV. Deglycosylation of PL and STBEV from $\mathrm{PE}$ showed that the $70 \mathrm{KDa}$ and the $50 \mathrm{KDa}$ bands were reduced to $48 \mathrm{KDa}$, suggesting glycosylation. Both $48 \mathrm{KDa}$ and $70 \mathrm{KDa}$ bands were subjected to MS, confirming Siglec-6 expression in both.

\section{DISCUSSION}

Our data shows that the inability to detect Siglec-6 in circulation might be due to the placenta secreting STBEV carrying a modified glycosylated form of Siglec-6 with a 70KDa molecular weight, significantly and uniquely upregulated in PE STBEV.

Keywords: Siglec-6, preeclampsia, glycosylation, extracellular vesicles (EV) 


\section{INTRODUCTION}

Preeclampsia (PE) is a disease affecting $2-8 \%$ of pregnancies worldwide [1] and remains a major cause of fetal and maternal morbidity and mortality, estimated to account for $15 \%$ of maternal deaths worldwide [2]. It is characterised, and currently primarily diagnosed, by newonset hypertension and proteinuria or other end organ dysfunction in the second half of pregnancy. The disease is progressive and can lead to complications such as maternal kidney and liver failure, intracranial haemorrhage, and death, as well as fetal growth restriction and many complications of prematurity.

The pathogenesis of PE is yet to be entirely elucidated, though it is widely believed that the placenta plays a pivotal role in the development of the syndrome and the only definite resolution of the disease is its delivery [3]. Among the pathogenetic characteristics of PE are failure to establish sufficient uteroplacental circulation, resulting in hypoxic conditions, and aggravated sterile maternal systemic inflammatory response, which trigger the release of proinflammatory, antiangiogenic and procoagulant factors by the placenta $[4,5]$. The syncytiotrophoblast (STB) layer of the placenta directly interfaces with the maternal circulation, into which it constitutively releases extracellular vesicles (EV). Syncytiotrophoblast-derived extracellular vesicles (STBEV) are potential carriers of these factors and have been suggested to be possible biomarkers and circulating biopsies of the placenta [6-9] and may have important signalling functions. STBEV are released in greater numbers in PE and have been implicated in the pathogenesis of the disease [10].

Siglecs (Sialic acid-binding immunoglobulin-like lectins) are a subgroup of the negatively charged carbohydrate-binding lectins that specifically bind to sialylated glycans in the pericellular matrix of all cell types [11] and are thought to be important for recognition of selfassociated glycans. They are transmembrane receptors that are predominantly expressed in cells of the immune system [12,13]. Several Siglecs, including siglec-6 have a cytosolic tyrosine-based motif that is inhibitory and regulates cellular activation and clonal expansion upon binding to the receptor [14-16]. Siglec-6 is expressed in the placenta $[17,18]$ and is upregulated in placental tissue and maternal blood in PE pregnancy [19-24]. Interestingly, although the protein is found in B-cells and mast cells in other species, the placental expression of Siglec-6 is human specific [25], making the elevated levels in PE all the more pertinent as $\mathrm{PE}$ is primarily postulated as a human disease [26]. 
Although Siglec-6 upregulation in placental tissue from PE patients was previously shown, its expression has not yet been examined in extracellular vesicles specifically. Is it possible that STBEV's derived from PE and normal pregnancy (NP) placentae express Siglec-6 in the same or a different form to that expressed by the Placenta? and what significance does this portray? Whilst undertaking this work we discovered a unique glycosylated form of Siglec-6 expressed on STBEVs which might explain the discrepancies in Siglec-6 detection in the circulation.

\section{METHODS}

\section{Human Subjects}

Ethical approval for this project was granted by the Central Oxfordshire Research Ethics Committee C (REFS 07/H0607/74 \& 07/H0606/148). Pregnant women undergoing elective caesarean sections prior to the onset of labor were selected prospectively from the maternity ward at the Women's Centre, John Radcliffe Hospital, Oxford. All participating women gave written, informed consent prior to the collection of their placentas. These were then collected and perfused within 10 minutes of delivery from women with NP and PE. NP was defined as a singleton pregnancy with no history of PE or hypertensive disorders. Patients with PE were defined according to the criteria of the International Society for the Study of Hypertension in Pregnancy as simultaneous debut of de novo hypertension (blood pressure $>140 / 90 \mathrm{mmHg}$ ) and proteinuria (>300 mg/day) after week 20 of gestation.

\section{Immunohistochemistry}

Immunohistochemistry analysis was performed as previously described [7]. Briefly, 8$\square \mathrm{m}$ formaldehyde fixed paraffin embedded (FFPE) tissue sections from NP were rehydrated and blocked in $10 \%$ fetal calf serum (FCS) in PBS supplemented with $1 \% 0.01 \mathrm{M}$ Tween-20 (PBS-T) for 1 hour. Tissue sections were incubated with polyclonal sheep anti-Siglec-6 (2 $\mu \mathrm{g} / \mathrm{ml}$ in $1 \% \mathrm{FCS}$ in PBS-T, AF2859, R\&D systems) at $4{ }^{\circ} \mathrm{C}$ overnight. Sections incubated with $1 \%$ FCS with no antibody served as the negative control. Sections were then incubated with HRP-conjugated secondary antibody anti-sheep (1/200 in $10 \%$ FCS in PBS-T, P0163, Dako Cytomation) for 1 hour at room temperature prior to visualisation with DAB (Vector laboratories) and counterstaining with haematoxylin (ThermoFisher). 
An ex vivo placental dual-lobe perfusion system [27], modified as previously described [28], and centrifugation were used to pellet and isolate whole STBEV preparations comprising of microvesicles (MV) and exosomes (EX).

\section{Immunoblotting}

Western blots were run under reducing conditions with the same protein mass $(20 \mu \mathrm{g}$, normalised based on bicinchoninic acid assay results) of either PL or STBEV samples. Membranes were blocked for $1 \mathrm{~h}$ with $5 \%$ Blotto (2BScientific) in $0.1 \%$ TBS-T before staining overnight with polyclonal Siglec-6 sheep $(0.8 \mu \mathrm{g} / \mathrm{ml}$, AF2859, R\&D systems) or loading control Actin antibody $(0.5 \mu \mathrm{g} / \mathrm{ml}$, A3853, Sigma Aldrich). Membranes were subsequently incubated with HRP-linked secondary antibodies for $1 \mathrm{~h}$ : anti-mouse $(1 / 5,000$, Cell Signalling Technology Inc.) and anti-sheep (1/4,000, P0163, Dako Cytomation) before treatment with enhanced chemiluminescence (ECL) substrates (Thermo Scientific) and exposure. PL immunoblot normalisation of protein expression was done against loading control and band intensities were calculated using ImageJ while for STBEV, we loaded the same mass of total protein.

\section{Deglycosylation}

PE PL and STBEV were deglycosylated using the Enzymatic Deglycosylation Kit for NLinked \& Simple O-linked Glycans (GK80110; Prozyme), following the manufacturer's 'Denaturing Protocol'. Following incubation with kit reagents, samples were boiled for 5 min and cooled to room temperature before $1 \mu \mathrm{l}$ each of deglycosylating enzymes N-Glycanase, Sialidase A, and O-Glycanase were added to each sample and incubated for 3 hours at $37{ }^{\circ} \mathrm{C}$. Glycosylated material was kept at $-20{ }^{\circ} \mathrm{C}$ until further use.

\section{Coomassie blue SDS-PAGE gel staining}

Protein bands in SDS-PAGE gels loaded with deglycosylated and untreated PE PL and STBEV (20 $\mu \mathrm{g} / \mathrm{sample}$ ) were visualised by staining with Coomassie blue dye. The gel was imaged and compared to an identical Western blot, then stored in $5 \% \mathrm{v} / \mathrm{v}$ glacial acetic acid at $4{ }^{\circ} \mathrm{C}$ until bands were excised under aseptic conditions. Cut out bands were stored at $-20{ }^{\circ} \mathrm{C}$ in $5 \% \mathrm{v} / \mathrm{v}$ 
acetic acid/ $/ \mathrm{dH}_{2} \mathrm{O}$. In-gel digestion and protein mass spectrometry were performed on excised gel pieces.

\section{Mass Spectrometry}

All proteomics and sample preparation was performed at the Discovery Proteomics Facility at the Target Discovery Institute, University of Oxford. Enriched EV samples were mixed with $100 \mathrm{uL}$ RIPA buffer (Pierce) and lysed on ice for 30min. Proteins were precipitated by methanol-chloroform precipitation. Proteins were digested with 200ng Trypsin (Promega) overnight at $37 \mathrm{C}$. Trypsin digestion was stopped by acidification to a final concentration of 1\% FA. Samples were desalted on Sola HRP SPE cartridges (Thermo Scientific) and dried down in a vacuum centrifuge. Peptides were reconstituted in loading buffer (2\% ACN/0.1\% TFA).

\section{LC-MS/MS}

Peptides were injected into a LC-MS system comprised of a Dionex Ultimate 3000 nano LC and a Thermo Q-Exactive. Peptides were separated on a 50-cm-long EasySpray column (ES803; Thermo Fisher) with a 75- $\mu$ m inner diameter and a 60 minute gradient of 2 to $35 \%$ acetonitrile in $0.1 \%$ formic acid and $5 \%$ DMSO at flow rate of $250 \mathrm{~nL} / \mathrm{min}$.

MS1 spectra were acquired with a resolution of 70,000 and AGC target of $3 \mathrm{e} 6$ ions for a maximum injection time of $100 \mathrm{~ms}$. The Top15 most abundant peaks were fragmented after isolation with a mass window of 1.6 That a resolution of 17,500 . The normalised collision energy was set to $28 \%$ (HCD).

Data was analysed with Progenesis QI (Waters). Raw data was imported using standard settings and manually refined retention time alignment. MS/MS data was searched in Mascot (Matrix Science) against a human database (fused Uniprot/Trembl, 03/2018) with carbamidomethylation (Cys) as fixed and Oxidation (Met)/Deamidation (Gln/Asn) as variable modifications. Precursor mass tolerance was set to $10 \mathrm{ppm}$ and fragment tolerance to $0.04 \mathrm{Da}$. Peptide identifications were FDR adjusted at $1 \%$, and identifications with Mascot score <20 were discarded. 


\section{Statistical Analysis}

Data was analysed using GraphPad Prism 8 software. Two-tailed, unpaired $t$-test and MannWhitney's test were used as appropriate based on the underlying distribution for comparison of patient characteristics. Values were presented as mean \pm standard error mean (SEM). Statistical analysis of immunoblot results was done with two-way ANOVA using multiple comparisons with Tukey correction. Statistical significance was defined as $\mathrm{p}<0.05$.

\section{RESULTS}

Siglec-6 protein levels are upregulated in placental tissue from preeclampsia patients

Siglec-6 has already been shown to be strongly expressed in PE placenta[22]. We confirmed strong Siglec-6 staining on NP placental tissue compared to IgG control (Figure 1A). Siglec-6 expression was consistent, localised on the syncytiotrophoblast layer of the chorionic villi, and confirmed previous findings [22,29]. The expression of Siglec-6 was corroborated by immunoblotting of NP and PE PL (Figure 1B). All samples displayed Siglec-6 expression in the form of the expected 50KDa bands, with a stronger signal in PE PL samples compared to NP (ns, Figure 1C). A previously undescribed 70KDa band was also observed when probing membranes for Siglec-6 and was strikingly more visible in PE PL compared to NP PL.

\section{KDa Siglec-6 band is upregulated on STBEV derived from PE}

Next, expression of Siglec-6 was investigated in STBEV derived from PE and NP placentae (Figure 2A). Immunoblotting revealed the absence of the expected 50KDa Siglec-6 band in both NP and PE STBEV. Instead, we observed again the 70KDa band, which was expressed at significantly higher levels in PE STBEV than NP STBEV ( $p<0.05$, Figure 2B). In general, as the gestational age increased, the intensity of the observed 70KDa Siglec-6 band on STBEV decreased. (Figure 2C).

70KDa Siglec-6 depleted following deglycosylation

We hypothesised that the 70KDa species seen in Siglec-6 immunoblotting of STBEV could be more heavily glycosylated. We tested this on PL, and STBEV derived from PE placentae using 
PNGase F, which specifically targets $\mathrm{N}$ - and O-linked glycosylation (Figure 3A). In both untreated (U) whole PL and STBEV, the 70KDa band was visible, whilst the 50KDa form was present only in PL. Following deglycosylation, the 70KDa band was no longer detectable by Western blot in PL and STBEV. Instead, 48KDa bands appeared in the deglycosylated PL and STBEV samples, yet the 50KDa band in the PL was still apparent after deglycosylation.

As the Siglec-6 70KDa band has not previously been described, we used mass spectrometry show whether or not this band was secondary to non-specific antibody binding, (Figure 3B). The 50KDa band is the established molecular weight of Siglec-6 and was therefore not subjected to mass spectrometry in the PE PL samples. The 70KDa bands in PE PL and STBEV and the $48 \mathrm{KDa}$ band that appeared in the deglycosylated STBEV samples were sent for analysis. The results of mass spectrometry confirmed the presence of Siglec-6 in the excised bands of size 70KDa prior to deglycosylation in both PL and STBEV, as well as in the 48KDa band post-deglycosylation in STBEV (Figure 3)

\section{DISCUSSION}

Siglec-6 is expressed in PE placentae at both transcript and protein levels [21,24,30-32]. Its expression does not vary by gestational age in both normal and preeclamptic placentas[33]. Our data shows that the expected Siglec-6 50KDa band is present in the PE and NP placental lysates, but absent in their respective STBEVs. Thus, our results revealed that the placenta does not secrete the expected 50KDa form of Siglec-6 via STBEV into the maternal circulation. Instead, STBEV seem to carry a glycosylated form of Siglec-6 (70KDa). We demonstrated that the $70 \mathrm{KDa}$ Siglec-6 form can be deglycosylated to an isoform of Siglec-6 with an approximate molecular mass of $48 \mathrm{KDa}$. The calculated mass of siglec-6 is approximately $48 \mathrm{KDa}$ (https://www.uniprot.org/uniprot/O43699) and the 48KDa band observed in our study may represent a native unmodified isoform of siglec- 6 while the $50 \mathrm{KDa}$ form may represent siglec6 with some form of posttranslational modification other than that targeted by the deglycosylation kit. Also, because the western blots were performed under a denaturing and reducing conditions, it is impossible for the $70 \mathrm{KDa}$ form to represent a glycosylation mediated protein-protein interaction. Protein mass spectrometry confirmed that the 70KDa band and deglycosylated 48KDa band on PE STBEV express the Siglec-6 protein. This prompts us to present a novel, N- or O-glycosylated form of Siglec-6 (70KDa) that is highly upregulated in PE PL and STBEV. 
Using Western blotting, a technique dependent on antibodies manufactured to be specific to known epitopes of a protein, Siglec-6 is present in placental tissue but not in maternal serum [21]. However, in proteomics studies, which use techniques such as protein mass spectrometry and RNA chip platform sequencing, Siglec-6 is detectable in both placental tissue [21,34] and maternal blood [24]. Such proteomics techniques lend themselves to a broader, non-epitopespecific detection of target proteins, which are useful in so far that they confirm Siglec-6 levels to be elevated in PE pregnancy, however, they do not distinguish epitopes or post-translational modifications. The difference between the 70KDa Siglec-6 form in placental lysates and STBEV derived from PE and NP is particularly striking and lends credence to the idea that there is a modified, glycosylated version of Siglec- 6 being exported from the placenta into the maternal circulation. We were looking for the expression of this novel version in the maternal circulation when the COVID19 pandemic closed the laboratories.

$\mathrm{PE}$ is a disease associated with an exaggerated maternal systemic inflammatory response compared to normal pregnancy [35]. Pathologic features of the PE syndrome such as endothelial and circulatory dysfunction are commonalities with inflammation, as are increased oxidative stress [36], complement system dysregulation and activation of both innate and adaptive immune system [37-39]. Aberrant glycosylation is known to pertain to the inflammatory response [40]. Glycosylation is a common post-translational modification of proteins that entails the enzymatic linking of oligosaccharides to newly synthesised proteins in the endoplasmic reticulum or the Golgi apparatus [41]. The specific glycan profile can reflect the tissue origin and biochemical condition in which the protein was produced [42]. Many major serum proteins such as $\operatorname{IgG}, \operatorname{IgA}$, transferrin, haptoglobin, alpha2-macroglobulin, and Creactive protein have been found to be glycosylated in inflammation. The glycosylated forms are able to form complexes with lectins, which have been found to be crucial to initiate the inflammatory response [43]. It is possible that a similar mechanism may be the case with Siglec-6 in PE pregnancies.

Glycosylated molecules are already used as biomarkers; glycated haemoglobin (HbA1c) is used for diagnosis of diabetes mellitus and is more reliable as a long term marker than fasting glucose [44]. We considered the possibility that the glycosylated forms observed may be because of gestational or pre-gestational diabetes in our sample population, but none of the patients whose samples were used had diabetes mellitus (data not shown). While we are yet to 
explore the functional significance of this, this interesting observation could reveal a role for the glycosylated form of Siglec-6 in PE pathology and, potentially, diagnostics.

In conclusion, our data shows that the placenta produces STBEVs that carry a modified glycosylated form of Siglec-6 with a $70 \mathrm{KDa}$ molecular weight which may explain the challenges associated with the detection of Siglec-6 in the circulation. Further investigations are underway to assess its role as a diagnostic tool.

\section{ACKNOWLEDGEMENTS}

We acknowledge the support of the National Institute of Health Research Clinical Research Network for assistance in patient recruitment and Fenella Roseman and Linda Holden, research midwives who kindly recruited the patients for this study.

\section{REFERENCES}

[1] S. Lisonkova, K.S. Joseph, Incidence of preeclampsia: risk factors and outcomes associated with early- versus late-onset disease, Am J Obs. Gynecol. 209 (2013) 544 e1-544 e12. https://doi.org/10.1016/j.ajog.2013.08.019.

[2] K.S. Khan, D. Wojdyla, L. Say, A.M. Gulmezoglu, P.F. Van Look, WHO analysis of causes of maternal death: a systematic review, Lancet. 367 (2006) 1066-1074. https://doi.org/10.1016/s0140-6736(06)68397-9.

[3] C. Redman, Pre-eclampsia: A complex and variable disease, Pregnancy Hypertens. 4 (2014) 241-242. https://doi.org/10.1016/j.preghy.2014.04.009.

[4] C.W. Redman, I.L. Sargent, Latest advances in understanding preeclampsia, Science (80-. ). 308 (2005) 1592-1594. https://doi.org/10.1126/science.1111726.

[5] S. Baumwell, S.A. Karumanchi, Pre-eclampsia: clinical manifestations and molecular mechanisms, Nephron Clin Pr. 106 (2007) c72-81. https://doi.org/10.1159/000101801.

[6] R.A. Dragovic, G.P. Collett, P. Hole, D.J.P. Ferguson, C.W. Redman, I.L. Sargent, D.S. Tannetta, Isolation of syncytiotrophoblast microvesicles and exosomes and their 
characterisation by multicolour flow cytometry and fluorescence Nanoparticle Tracking Analysis, Methods. (2015). https://doi.org/10.1016/j.ymeth.2015.03.028.

[7] C. Motta-Mejia, N. Kandzija, W. Zhang, V. Mhlomi, A.S. Cerdeira, A. Burdujan, D. Tannetta, R. Dragovic, I.L. Sargent, C.W. Redman, U. Kishore, M. Vatish, Placental Vesicles Carry Active Endothelial Nitric Oxide Synthase and Their Activity is Reduced in Preeclampsia, Hypertension. 70 (2017) 372-381. https://doi.org/10.1161/hypertensionaha.117.09321.

[8] G.P. Collett, C.W. Redman, I.L. Sargent, M. Vatish, Endoplasmic reticulum stress stimulates the release of extracellular vesicles carrying danger-associated molecular pattern (DAMP) molecules, Oncotarget. 9 (2018) 6707-6717. https://doi.org/10.18632/oncotarget.24158.

[9] D. Tannetta, G. Collett, M. Vatish, C. Redman, I. Sargent, Syncytiotrophoblast extracellular vesicles - Circulating biopsies reflecting placental health, Placenta. 52 (2017) 134-138. https://doi.org/10.1016/j.placenta.2016.11.008.

[10] S.I. Gilani, T.L. Weissgerber, V.D. Garovic, M. Jayachandran, Preeclampsia and Extracellular Vesicles, Curr Hypertens Rep. 18 (2016) 68. https://doi.org/10.1007/s11906-016-0678-x.

[11] S. Kelm, A. Pelz, R. Schauer, M.T. Filbin, S. Tang, M.E. de Bellard, R.L. Schnaar, J.A. Mahoney, A. Hartnell, P. Bradfield, et al., Sialoadhesin, myelin-associated glycoprotein and CD22 define a new family of sialic acid-dependent adhesion molecules of the immunoglobulin superfamily, Curr Biol. 4 (1994) 965-972.

[12] M.S. Macauley, P.R. Crocker, J.C. Paulson, Siglec-mediated regulation of immune cell function in disease, Nat Rev Immunol. 14 (2014) 653-666. https://doi.org/10.1038/nri3737.

[13] S. von Gunten, B.S. Bochner, Basic and clinical immunology of Siglecs, Ann N Y Acad Sci. 1143 (2008) 61-82. https://doi.org/10.1196/annals.1443.011.

[14] M. Zhang, T. Angata, J.Y. Cho, M. Miller, D.H. Broide, A. Varki, Defining the in vivo function of Siglec-F, a CD33-related Siglec expressed on mouse eosinophils, Blood. 109 (2007) 4280-4287. https://doi.org/10.1182/blood-2006-08-039255. 
[15] P.R. Crocker, J.C. Paulson, A. Varki, Siglecs and their roles in the immune system, Nat Rev Immunol. 7 (2007) 255-266. https://doi.org/10.1038/nri2056.

[16] J. V Ravetch, L.L. Lanier, Immune inhibitory receptors, Science (80-. ). 290 (2000) 84-89. https://doi.org/10.1126/science.290.5489.84.

[17] E.C. Brinkman-Van der Linden, N. Hurtado-Ziola, T. Hayakawa, L. Wiggleton, K. Benirschke, A. Varki, N. Varki, Human-specific expression of Siglec-6 in the placenta, Glycobiology. 17 (2007) 922-931. https://doi.org/10.1093/glycob/cwm065.

[18] Y. Takei, S. Sasaki, T. Fujiwara, E. Takahashi, T. Muto, Y. Nakamura, Molecular cloning of a novel gene similar to myeloid antigen CD33 and its specific expression in placenta, Cytogenet Cell Genet. 78 (1997) 295-300. https://doi.org/10.1159/000134676.

[19] V. Sitras, R.H. Paulssen, H. Gronaas, J. Leirvik, T.A. Hanssen, A. Vartun, G. Acharya, Differential placental gene expression in severe preeclampsia, Placenta. 30 (2009) 424-433. https://doi.org/10.1016/j.placenta.2009.01.012.

[20] S. Tsai, N.E. Hardison, A.H. James, A.A. Motsinger-Reif, S.R. Bischoff, B.H. Thames, J.A. Piedrahita, Transcriptional profiling of human placentas from pregnancies complicated by preeclampsia reveals disregulation of sialic acid acetylesterase and immune signalling pathways, Placenta. 32 (2011) 175-182. https://doi.org/10.1016/j.placenta.2010.11.014.

[21] K.K. Rumer, J. Uyenishi, M.C. Hoffman, B.M. Fisher, V.D. Winn, Siglec-6 expression is increased in placentas from pregnancies complicated by preterm preeclampsia, Reprod Sci. 20 (2013) 646-653. https://doi.org/10.1177/1933719112461185.

[22] V.D. Winn, M. Gormley, A.C. Paquet, K. Kjaer-Sorensen, A. Kramer, K.K. Rumer, R. Haimov-Kochman, R.-F. Yeh, M.T. Overgaard, A. Varki, C. Oxvig, S.J. Fisher, Severe preeclampsia-related changes in gene expression at the maternal-fetal interface include sialic acid-binding immunoglobulin-like lectin-6 and pappalysin-2., Endocrinology. 150 (2009) 452-462. https://doi.org/10.1210/en.2008-0990.

[23] D.A. Enquobahrie, M. Meller, K. Rice, B.M. Psaty, D.S. Siscovick, M.A. Williams, Differential placental gene expression in preeclampsia, Am J Obs. Gynecol. 199 
(2008) 566 e1-11. https://doi.org/10.1016/j.ajog.2008.04.020.

[24] O. Erez, R. Romero, E. Maymon, P. Chaemsaithong, B. Done, P. Pacora, B. Panaitescu, T. Chaiworapongsa, S.S. Hassan, A.L. Tarca, The prediction of late-onset preeclampsia: Results from a longitudinal proteomics study, PLoS One. 12 (2017) e0181468. https://doi.org/10.1371/journal.pone.0181468.

[25] H. Yokoi, A. Myers, K. Matsumoto, P.R. Crocker, H. Saito, B.S. Bochner, Alteration and acquisition of Siglecs during in vitro maturation of CD34+ progenitors into human mast cells, Allergy. 61 (2006) 769-776. https://doi.org/10.1111/j.13989995.2006.01133.x.

[26] R.A. Chez, Nonhuman primate models of toxemia of pregnancy, Perspect Nephrol Hypertens. 5 (1976) 421-424.

[27] R.A. Dragovic, J.H. Southcombe, D.S. Tannetta, C.W. Redman, I.L. Sargent, Multicolor flow cytometry and nanoparticle tracking analysis of extracellular vesicles in the plasma of normal pregnant and pre-eclamptic women, Biol Reprod. 89 (2013) 151. https://doi.org/10.1095/biolreprod.113.113266.

[28] R.A. Dragovic, C. Gardiner, A.S. Brooks, D.S. Tannetta, D.J. Ferguson, P. Hole, B. Carr, C.W. Redman, A.L. Harris, P.J. Dobson, P. Harrison, I.L. Sargent, Sizing and phenotyping of cellular vesicles using Nanoparticle Tracking Analysis, Nanomedicine. 7 (2011) 780-788. https://doi.org/10.1016/j.nano.2011.04.003.

[29] K.K. Rumer, M.D. Post, R.S. Larivee, M. Zink, J. Uyenishi, A. Kramer, D. Teoh, K. Bogart, V.D. Winn, Siglec-6 is expressed in gestational trophoblastic disease and affects proliferation, apoptosis and invasion., Endocr. Relat. Cancer. 19 (2012) 827840. https://doi.org/10.1530/ERC-11-0379.

[30] E.A. Trifonova, T. V Gabidulina, N.I. Ershov, V.N. Serebrova, A.Y. Vorozhishcheva, V.A. Stepanov, Analysis of the placental tissue transcriptome of normal and preeclampsia complicated pregnancies, Acta Naturae. 6 (2014) 71-83.

[31] J.H. Kang, H. Song, J.A. Yoon, D.Y. Park, S.H. Kim, K.J. Lee, A. Farina, Y.K. Cho, Y.N. Kim, S.W. Park, G.J. Kim, S.H. Shim, D.H. Cha, Preeclampsia leads to dysregulation of various signaling pathways in placenta, J Hypertens. 29 (2011) 928- 
936. https://doi.org/10.1097/HJH.0b013e328344a82c.

[32] T. Kaartokallio, A. Cervera, A. Kyll nen, K. Laivuori, J. Kere, H. Laivuori, F.C.I. Group, Gene expression profiling of pre-eclamptic placentae by RNA sequencing, Sci Rep. 5 (2015) 14107. https://doi.org/10.1038/srep14107.

[33] K.K. Rumer, J. Uyenishi, M.C. Hoffman, B.M. Fisher, V.D. Winn, Siglec-6 expression is increased in placentas from pregnancies complicated by preterm preeclampsia, Reprod. Sci. (2013). https://doi.org/10.1177/1933719112461185.

[34] V.D. Winn, M. Gormley, S.J. Fisher, The Impact of Preeclampsia on Gene Expression at the Maternal-Fetal Interface, Pregnancy Hypertens. 1 (2011) 100-108. https://doi.org/10.1016/j.preghy.2010.12.001.

[35] C.W. Redman, I.L. Sargent, Pre-eclampsia, the placenta and the maternal systemic inflammatory response--a review, Placenta. 24 Suppl A (2003) S21-7.

[36] C.A. Hubel, Dyslipidemia, iron, and oxidative stress in preeclampsia: assessment of maternal and feto-placental interactions, Semin Reprod Endocrinol. 16 (1998) 75-92. https://doi.org/10.1055/s-2007-1016255.

[37] J.F. Regal, R.M. Burwick, S.D. Fleming, The Complement System and Preeclampsia, Curr Hypertens Rep. 19 (2017) 87. https://doi.org/10.1007/s11906-017-0784-4.

[38] E.B. Taylor, J.M. Sasser, Natural killer cells and T lymphocytes in pregnancy and preeclampsia, in: Clin Sci, 2017 The Author(s). Published by Portland Press Limited on behalf of the Biochemical Society., Department of Physiology and Biophysics, University of Mississippi Medical Center, 2500 North State Street Jackson, MS 39216, U.S.A. ertaylor@umc.edu. Department of Pharmacology and Toxicology, University of Mississippi Medical Center, 2500 North State St, 2017: pp. 2911-2917. https://doi.org/10.1042/cs20171070.

[39] C.A. Lok, J. Jebbink, R. Nieuwland, M.M. Faas, K. Boer, A. Sturk, J.A. Van Der Post, Leukocyte activation and circulating leukocyte-derived microparticles in preeclampsia, Am J Reprod Immunol. 61 (2009) 346-359. https://doi.org/10.1111/j.16000897.2009.00701.x. 
[40] O. Gornik, G. Lauc, Glycosylation of serum proteins in inflammatory diseases, Dis Markers. 25 (2008) 267-278. https://doi.org/10.1155/2008/493289.

[41] R.G. Spiro, Protein glycosylation: nature, distribution, enzymatic formation, and disease implications of glycopeptide bonds, Glycobiology. 12 (2002) 43R-56R. https://doi.org/10.1093/glycob/12.4.43r.

[42] G. Lauc, J. Kristic, V. Zoldos, Glycans - the third revolution in evolution, Front Genet. 5 (2014) 145. https://doi.org/10.3389/fgene.2014.00145.

[43] S.R. Barthel, J.D. Gavino, L. Descheny, C.J. Dimitroff, Targeting selectins and selectin ligands in inflammation and cancer, Expert Opin Ther Targets. 11 (2007) 1473-1491. https://doi.org/10.1517/14728222.11.11.1473.

[44] S.I. Sherwani, H.A. Khan, A. Ekhzaimy, A. Masood, M.K. Sakharkar, Significance of HbA1c Test in Diagnosis and Prognosis of Diabetic Patients, Biomark Insights. 11 (2016) 95-104. https://doi.org/10.4137/bmi.s38440.

Table 1. Clinical data of human subjects whose placentas were used for isolation of STBEV. Data presented as Mean \pm standard error mean (SEM), significant difference shown as $\mathrm{p}<0.0001(* * * *)$ or not significant (ns).

Figure 1. Siglec-6 expression in placental lysate. A) Representative immunohistochemistry staining of Siglec-6 expressed on the syncytiotrophoblast layer of NP placental tissue, and negative control. Scale bar is $200 \mu \mathrm{m}$. B) Western blot of whole placental lysates of PE $(n=7)$ and NP pregnancies $(n=7)$. The PE samples are in order of advancing gestation (weeks) while the NP samples are not as it is impossible to obtain 'normal' samples less than 37 gestational age (weeks). Siglec-6 (50KDa) was absent from STBEV derived from both NP and PE placentae. Abundant expression of Siglec-6 at 50KDa band in PE and NP placental lysates, and observation of a 70KDa band mainly expressed in the PE samples. C) Protein normalization against Actin indicated upregulation of the Siglec-6 (50KDa) in PE subjects (not significant, ns). Error bars illustrate standard error mean (SEM). 
Figure 2. Upregulated and specific expression of $70 \mathrm{KDa}$ form of Siglec-6 on STBEV derived from PE placentas. A) Western blot analysis of Siglec-6 expression on STBEV from NP $(n=11)$, and PE $(n=11)$ placentae. The observed 70KDa band was instead present in PE and NP STBEV. B) Normalized densitometry of the 70KDa bands in PE and NP showed significant upregulation in PE $(\mathrm{p}<0.05)$. C) Normalized densitometry of the 70KDa bands in PE STBEV $(n=1$ per gestational age $)$ against placental delivery gestation showed a negative correlation, suggesting decreasing expression of the Siglec-6 (70KDa) as gestation advances.

Figure 3. The effect of deglycosylation on placental and STBEV Siglec-6 forms. A) Representative immunoblotting of Siglec-6 expression on Preeclamptic PL and STBEV, showing untreated PL and STBEV (U), and PL and STBEV that underwent deglycosylation (D). Upon deglycosylation the 70KDa form was missing from both PL and STBEV, instead being replaced by a previously not seen $\sim 48 \mathrm{KDa}$ form of Siglec-6. B) Coomassie Blue staining of a SDS gel with the same set up as in Figure 3a C) Mass spectrometry analysis result table of excised bands confirming the presence of Siglec-6 at $\sim 48 \mathrm{KDa}$ and $70 \mathrm{KD}$ a protein bands. 